\title{
Andropenis $®$ Plus Oral Therapy (Peironiemev) Versus Intralesional Verpamil in the Treatment of the Acute Phase of Peyronie's Disease
}

\section{Pisano Francesca ${ }^{*}$, Falcone Marco ${ }^{1}$, Di Marco Massimiliano ${ }^{2}$, Giubilei Gianluca ${ }^{3}$, Soria Francesco ${ }^{1}$, Fiorito Chiara $^{1}$,} Bartoletti Riccardo ${ }^{4}$, Pastore Antonio Luigi ${ }^{5}$, Tizzani Alessandro ${ }^{1}$, Mondaini Nicola ${ }^{6}$ and Gontero Paolo ${ }^{1}$

${ }^{1}$ Department of Urology, University of Turin, Italy

${ }^{2}$ Department of Urology, Guarnieri Hospital, Italy

${ }^{3}$ Department of Urology, Leonardo Da Vinci Hospital, Italy

${ }^{4}$ Universita Degli Studi di Firenze, Urology, Italy

${ }^{5}$ Department of Medico-Surgical Sciences and Biotechnologies, Sapienza University of Rome, Italy

${ }^{6}$ Department of Urology, Santa Maria Annunziata Hospital, Italy

*Corresponding author: Francesca Pisano, MD, Department of Urology, City of Health and Science-Molinette Hospital, University of Turin, Corso Bramante 88, 10126 Torino, Italy.

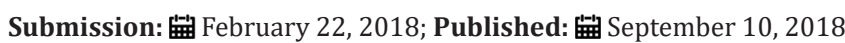

\begin{abstract}
Introduction: The aim of this study was to compare the association of a POTABA based oral compound (PeironiMEV®) with a penile extender (Andropenis $($ ) stretching protocol to Verapamil injections in the treatment of Peyronie's disease (PD).

Materials and methods: Patients with PD naive to any treatment were enrolled and randomized: Andropenis 8 hours per day plus PeironiMEV 2 tablets/die for 6 months (arm 1); Intralesional Verapamil $5 \mathrm{mg} / 2 \mathrm{ml}$ every two weeks for 6 months (arm 2). Patients were evaluated at 3,6 , 12 and 24 months after baseline. Plaque size, curvature degree and penile lenght were recorded at each visit during the follow-up. Functional results were assessed through: Erectile Function (EF) domain score of the International Index of Sexual Function (IIEF), Sexual Encounter Profile (SEP 2) and VAS score.
\end{abstract}

Results: 40 patients were enrolled. At 12 months, VAS and EF domains of IIEF improvement was significantly higher in arm 1 (p<0.001). In both arms penile curvature showed a significant reduction $(\mathrm{p}<0.001)$ at 12 months follow up. Penile flaccid and stretched length had a trend to improvement in arm 1 (p 0.087 and 0.078 respectively).

Conclusion: PeironiMEV plus penile stretching impacted on patient erectile function. Moreover, it demonstrated to be more effective than verapamil injections in pain control.

Keywords: Peyronie disease; Penile extender; Conservative treatments; Verapamile injection; Penile curvature

\section{Introduction}

Peyronie's disease (PD) is characterized by an inflammatory response of the tunica albuginea with fibroblast proliferation leading to the formation of a fibrous plaque causing a retracting scarring process. It's well known that its clinical syndrome is characterized by at least one of the present symptoms: painful erections, erectile dysfunction or penile deformities [1]. PD can be treated either with a conservative or a surgical approach. The first line treatments, to be mostly used in acute settings, are either the medical or the physical treatment as for the EAU guidelines [2]. On the other hand, surgical therapy is an effective option to be reserved for a stable disease when either the penile deformation or the erectile dysfunctions dramatically impair patients' sexual activity [2].

The medical treatment can be either oral or injective. Among the various oral compounds proposed for PD, POTABA has shown promising efficacy $[3,4]$. Among the injective therapies, intralesional Verapamil is one of the most popular for PD. This treatment has shown some efficacy on pain relief, plaque reduction and curvature decrease but with a low level of evidence [5]. Recently, penile extenders have been offered to PD patients with notable improvements on penile curvature in up to $50 \%$ of cases. Moreover, some investigators had claimed the extender devices to 
produce a significant lengthening of the shaft thus contrasting the shortening effect provoked by the fibrous plaque [6]. The aim of our study was to compare for the first time in the scientific literature the clinical outcomes of a combination of a penile traction therapy and a POTABA based compound oral assumption and an intralesional Verapamil injection for PD in acute phase.

\section{Materials and Methods}

\section{Study design and objectives}

Patients with a new onset clinical diagnosis of PD were considered eligible in the study. The clinical diagnosis was confirmed when three or more of the following elements where noticed: penile pain, palpable penile plaque, penile curvature, penile shortening or erectile dysfunction. The following represented exclusion criteria: a stable chronic phase of the disease, a previous use of any medical or physical therapy and the inability to comply with either of the proposed treatments, the presence of any penile deformities, the use of phosphodiesterase 5 inhibitors. Changes in penile curvature and sexual function after 6 months of treatment were considered primary study end points. Whereas penile length, penile girth and plaque size were set a secondary end point. The study was conducted according to the guidelines and principles of the Declaration of Helsinki and standard ethical conduct for research involving humans. All enrolled subjects provided written informed consent for study participation. The present study was approved by the local ethical committee.

\section{Treatment Arms}

Patients were randomized according to a computergenerated random sequence in two different groups using www. randomization.com

\section{Arm 1}

Andropenis ${ }^{\circledR}$ for 8 hours/die plus PeironiMEV® 2 tablets/ die for six months. Andropenis $\AA$ (Andromedical, Madrid, Spain) is a device designed to exert a continuous and gradually increasing traction force on the penis. It consists of a plastic ring, where the penis is introduced and from where two dynamic metallic rods originate the traction. PeyronieMEV® composition consisted of $36 \mathrm{mg}$ vitamin E, $100 \mathrm{mg}$ para-aminobenzoic acid, $100 \mathrm{mg}$ propolis, $80 \mathrm{mg}$ blueberry anthocyanins, $50 \mathrm{mg}$ Soja isoflavones, $25 \mathrm{mg}$ Muria Pauma, 25mg Damiana and 50mg Persea Americana.

\section{Results}

Table 1: Baseline patients' characteristics.

\section{Arm 2}

Intralesional injections of Verapamil $10 \mathrm{mg} / 2 \mathrm{cc}$ every 2 weeks for 6 months. Patients received a local anesthesia with topic $2.5 \%$ lidocaine, given 30 minutes before the treatment. Injections were administered in the tunica albuginea surrounding the plaque, carefully avoiding injuries to urethra ventrally and the neurovascular bundle dorsally.

\section{Baseline and Follow-up Investigations}

A full medical and sexual history and a complete physical examination were recorded during the first outpatient's clinic visit. The Erectile Function (EF) domain score of the International Index of Sexual Function (IIEF), the Sexual Encounter Profile question 2 (SEP 2) and the Visual Analogue Scale (VAS) were administrated at the baseline and at the follow up visit at 1, 3, 6 and 12 months. All patients underwent a penile dynamic Doppler ultrasonography in order to assess the size and the location of the plaques and to rule out the presence of any calcification of the tunica albuginea. The degree of curvature was evaluated at the maximum erection state after alprostadil intracavernous injection through photographic pictures taken by the physician from three angles (frontal, lateral and dorsal). The amplitude of the curvature was determined by placing a goniometer in the angle formed by the intersection of two drawn segments running parallel to each of the two-banded portions of the shafts. Penile length and penile girth were measured in both the flaccid and the erect state using a taper ruler. The aforementioned measures were detected at the baseline and at the follow up visits at 1, 3, 6 and 12 months.

\section{Statistics}

Data are presented as mean (standard deviation) or median (inter-quartile range). Comparison between and within groups was made using the unpaired t-test and paired t-test for continuous variables respectively. Chi-square was used for the categorical variables. All tests were 2-tailed. To test for a significant difference in means over time we performed an ANOVA analysis for repeated measures. The level of significance was set at 0.05 . In a previous observational study, we found a mean reduction in penile curvature of $7^{\circ}$ with standard deviation of 3.2 [6]. Based on this, the numerosity of the study was set at 40 patients to demonstrate a mean of $10^{\circ}$ in patients with PD with a 5\% risk of type I error and power of $90 \%$. Analysis was conducted using the SAS software package (SAS Institute, Cary, NC; version 8.02).

\begin{tabular}{|c|c|c|c|}
\hline Variables Analyzed & ARM 1 & ARM 2 & p Value \\
\hline Age & $55(41-74)$ & $58(47-78$ & NS \\
\hline Mean Duration Disease (SD) & $8.1(5.8)$ & $7.3(3.1)$ & NS \\
\hline $\begin{array}{c}\text { Mean penile length } \\
\text { - Flaccid (SD) } \\
\text { - Stretched (SD) }\end{array}$ & $8.6(2.2)$ & $7.8(1.4)$ & NS \\
\hline Mean Curvature Degree & $11.1(2.5)$ & $10.2(2.5)$ & NS \\
\hline
\end{tabular}




\begin{tabular}{|c|c|c|c|}
\hline Mean Penile Girth & $10.6(2.1)$ & $9.2(0.7)$ & $11.3(8.2)$ \\
\hline Mean Plaque Size & $11.5(6.8)$ & $1.7(2.1)$ & NS \\
\hline Mean VAS & $3.3(3.2)$ & $17.6(9)$ & NS \\
\hline Mean IIEF 5 & $18.1(6.5)$ & $3.7(1.9)$ & NS \\
\hline Mean SEP & $3.1(1.7)$ & NS \\
\hline
\end{tabular}

40 patients with a diagnosis of PD met the inclusion criteria and entered the study. They were randomized in the treatment arms already described above. Baseline patients' characteristics are listed in Table 1. No differences were recorded in the two groups at the baseline. All patients completed the study protocol. Results are summarized in Table 2. In both arms 1 and 2 penile curvature showed a statistically significant reduction $(p=0.001$ and $p=0.04$, respectively) at 12 months follow up (Figure 1). Penile pain, assessed through the VAS score, had a significant reduction in arm 1 compared to arm 2 ( $p<0.01$ ) (Figure 2). As for the erectile function, at 12 months of follow-up, IIEF score showed a statistically significant improvement in the arm 1, from a mean baseline of 18.1 (SD 6.5) to 19.5 (SD 5.6) with a p value $<0.01$ (Figure 3). Penile flaccid and stretched length had a trend to improvement in arm 1 after 12 months follow-up (p 0.087 and p 0.078 respectively). Additionally, no significant differences were observed in penile girth, plaque size and SEP score in both arms. Two patients treated with Verapamil injections reported side effects, a penile hematoma and local erythema.

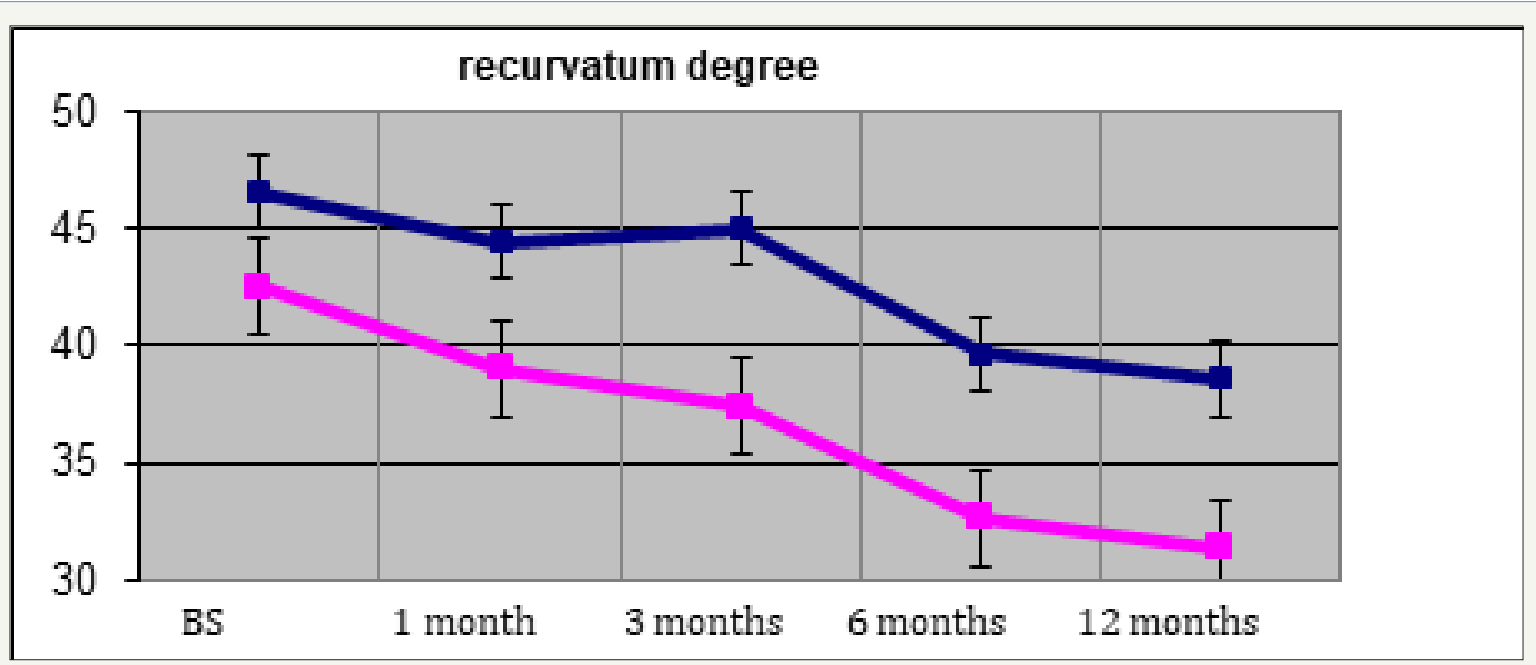

Figure 1: Changes in penile curvature after 1,3 and 6 months treatment and at 12 months (after 6 months of treatment discontinuation) in both arms.

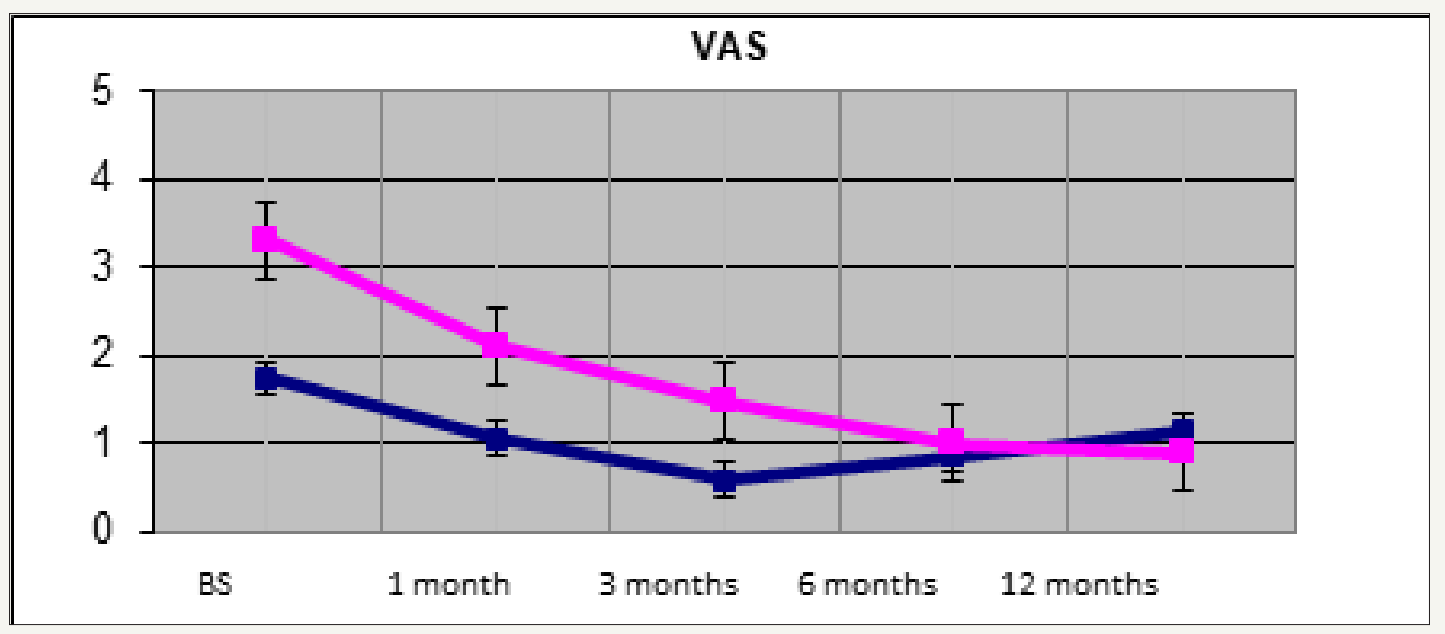

Figure 2: Pain relief after 1,3 and 6 months treatment and at 12 months (after 6 months of treatment discontinuation) in both arms. 


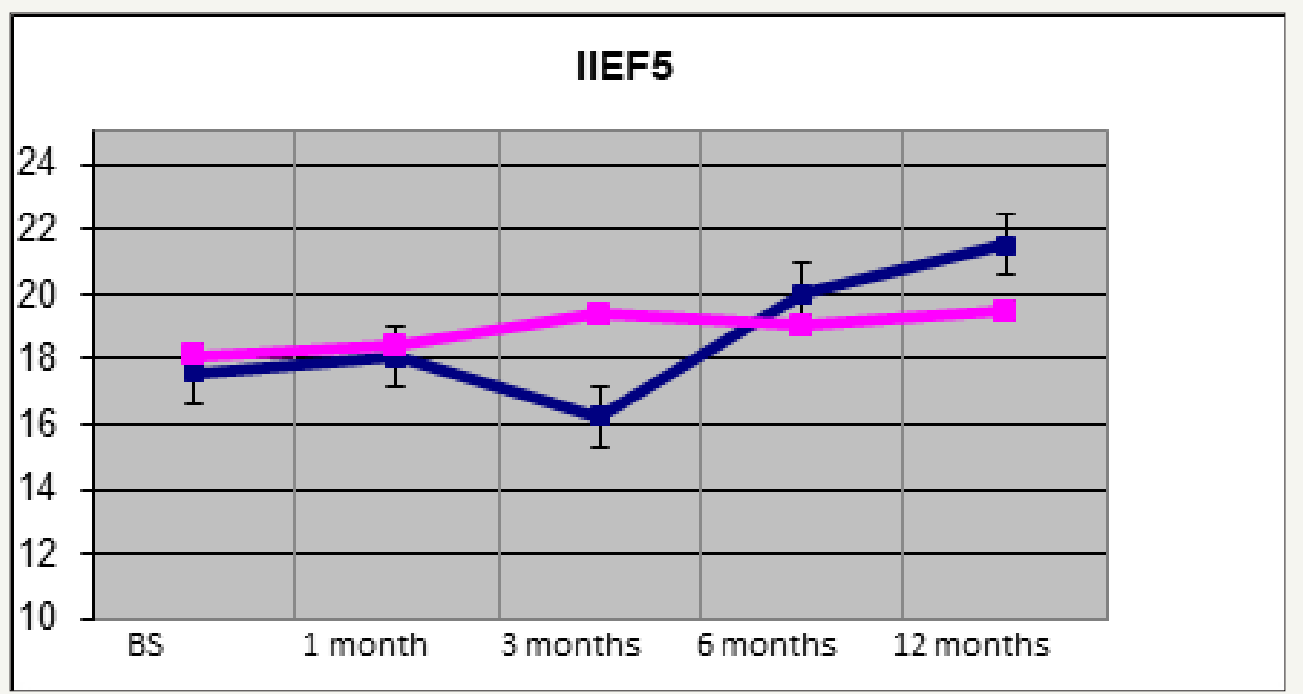

Figure 3: Improve of erectile function after 1,3 and 6 months treatment and at 12 months (after 6 months of treatment discontinuation) in both arms.

Table 2: Mean changes in the outcome measure at 12 months in both groups.

\begin{tabular}{|c|c|c|c|c|c|c|}
\hline \multirow{2}{*}{ Variables Analyzed } & \multicolumn{3}{|c|}{ ARM 1 } & \multicolumn{2}{c|}{ ARM 2 } \\
\cline { 2 - 6 } & Baseline & $\mathbf{1 2 \text { months }}$ & p value & Baseline & 12 months & p value \\
\hline $\begin{array}{c}\text { Mean Penile Length } \\
\text { - Flaccid (SD) } \\
\text { - Stretched (SD) }\end{array}$ & $\begin{array}{c}8.6(2.2) \\
11.1(2.5)\end{array}$ & $\begin{array}{c}9.5(2.7) \\
11.6(2.8)\end{array}$ & $\begin{array}{c}\text { NS } \\
\text { NS }\end{array}$ & $\begin{array}{c}7.8(1.4) \\
10.2(2.5)\end{array}$ & $\begin{array}{c}8.2(2.1) \\
10.9(1.9) \\
\text { NS }\end{array}$ \\
\hline Mean Curvature Degree & $42.5(18.1)$ & $31.3(21.6)$ & 0.001 & $46.5(18.5)$ & $31.5(10.4)$ & 0.04 \\
\hline Mean Penile Girth & $10.6(2.1)$ & $10.8(2)$ & NS & $9.2(0.7)$ & $9.8(0.7)$ & NS \\
\hline Mean Plaque Size & $11.5(6.8)$ & $10.2(5.2)$ & NS & $11.3(8.2)$ & $8.4(4.5)$ & NS \\
\hline VAS & $3.3(3.2)$ & $0.9(1.4)$ & $<0.01$ & $1.7(2.1)$ & $1.1(1.8)$ & NS \\
\hline IIEF & $18.1(6.5)$ & $19.5(5.6)$ & $<0.01$ & $17.6(9)$ & $21.5(2.6)$ & NS \\
\hline SEP & $3.1(1.7)$ & $3.7(1.9)$ & NS & $3.7(1.9)$ & $3.3(2.2)$ & NS \\
\hline
\end{tabular}

\section{Discussion}

Peyronie's disease represents a quite common disorder with prevalence rates ranging from 0.4 to $9 \%$ among adult males [2]. Conservative treatments are especially focused on patients in the early stage of this pathological condition, while their role in men with stable disease has not been adequately defined $[7,8]$. In spite of the numerous treatment options described, it still remains a challenging clinical problem in sexual medicine with lack of effective medical remedies [9]. Moreover, the results of the studies on conservative treatments for Peyronie's disease are often contradictory making it difficult to individuate the best approach in everyday-practice [8]. It is widely accepted that the medical approach, in terms of oral compounds, injective drugs or physical therapies remains controversial [10]. A recent not-randomized retrospective analysis aiming to compare the effect on penile curvature following the addition of a penile traction therapy to Verapamil injections versus the use of intralesional Verapamil alone failed to show any significance [11].
The lack of knowledge on the pathogenesis of PD represents one of the key elements that preclude the development of appropriate therapeutic strategies. Nevertheless, the chronic phase of PD is characterized by a strong fibrosis and no conservative treatments have been shown to provide some useful results in terms of penile curvature reduction and erectile function improvement [7]. Penile length loss represents a common consequence of the fibrotic retraction secondary to $\mathrm{PD}$ and it has been clearly showed to have a detrimental effect on the quality of sexual life [11]. In this context, the penile traction devices were proposed as a treatment option. The rationale of this approach derived from the evidence that several signaling cascades are activated by the tension [12]. Therefore, penile extenders have been thought to induce a lengthening of the shaft thus minimizing the shortening effect provoked by fibrous plaques [6]. Conforming to some investigators, in our population there was a trend toward measured length improvement. The reduction of penile curvature was probably the most notable clinical finding of our study. At the same time, for the penile length 
a trend improvement was pointed out. However, according to our experience and to the evidences of the literature, any gain in penile length, even if not statistically significant, may reinforce patient's satisfaction and self-confidence [13].

Martinez et al. [14] in a recent study described the penile traction therapy as an effective treatment for the acute phase of the Peyronie's disease in terms of pain reduction, penile curvature decreases and improvement in sexual function. Our results support these findings showing a significant improvement of both the curvature and the erection related pain.

To date there is still a lack of an international consensus on the most effective medical treatment for Peyronie's disease. Vitamin E is commonly prescribed by the majority of the andrologists, however a double blind, placebo-controlled crossover study failed to show a significant effect both on penile deformity and plaque size reduction [15]. In our study the POTABA and vitamin E based compound was administered according to the poor literature evidences. POTABA is the only antioxidant agent approved as "possibly effective" by the food and Drug Administration in the treatment of IPP [2]. It has shown some promising efficacy in pain relief [3] and plaque size reduction [4]. As shown by the literature, POTABA play a role in the reduction of plaque size [4]. Unfortunately, there's still a lack of evidence about the efficacy of this oral compound on the penile curvature. Abern et al. [11] in a non-randomized retrospective analysis showed only not statistically significant subjective improvements in curvature following the addition of a penile traction therapy to Verapamil injections versus the use of intralesional Verapamil alone. However, at long-term followup, the association therapy demonstrated to be more effective on the quality of the erectile function, compared to the verapamil injections alone. This result can be explained by a synergic effect of the two different therapies that equally contributes to the symptoms reliefs. Based on this evidence, in the current study, we aimed to increase the treatment outcome in terms penile curvature and pain of the POTABA based compounds, adding in association a penile extender device.

Focusing on our study, it is difficult to assess the mechanism underlying the notable results in terms of improvement of sexual function. Surely, the reduction of pain during sexual intercourses could represent a significant factor sustaining the improvement of sexual function. As well as the reduction of penile curvature that yields in easy penetrative intercourses and a gain in self-confidence. Regarding plaque size, neither Verapamil nor traction therapy showed a positive effect. However, no actual scientific evidences have ever demonstrated a clear relation between size of the plaque, curvature and quality of erections.

Our study underlines the positive effects of the combination of physical plus oral therapy in patients affected by Peyronie's disease. The combination therapy reported good results in terms of penile curvature and erectile function. Besides this combination treatment is less invasive compared to the injection therapy. In order to claim the combination of the physical and the oral therapy to be a validated approach to treat PD, our data should be validated through larger prospective studies.

\section{Conclusion}

The association of penile extender (Andropenis) plus oral treatment (PeironiMEV) produces a significant improvement of both the penile curvature and the erectile function when compared with Verapamil injection therapy alone. None the last, a significant reduction of the erection related pain was also recorded in the combination treatment arm.

\section{Conflict of Interest}

All authors certify that all conflicts of interest, including specific financial interests and relationships and affiliations relevant to the subject matter or materials discussed in the manuscript (e.g., employment/affiliation, grants or funding, consultancies, honoraria, stock ownership or options, expert testimony, royalties, or patents filed, received, or pending), are the following: None.

\section{Authors' Contribution}

All authors have contributed equally to the drafting of the manuscript. All authors read and approved the final version of the manuscript.

\section{Acknowledgement}

The authors declare that the development of the manuscript was not supported by an honorarium, a grant, or any other sources of support, including sponsorship or any material sources of support.

\section{References}

1. Bella AJ, Perelman MA, Brant WO, Lue TF (2007) Peyronie's disease (CME). J Sex Med 4(6): 1527-1538.

2. Hatzimouratidis K, Eardley I, Giuliano F, Hatzichristou D, Moncada I, et al. (2012) EAU guidelines on penile curavture. Eur Urol 62: 543-552.

3. Shah PJR, Green NA, Adib RS, Hamilton SPA, Smith P, et al. (1983) A multicentre double-blind controlled clinical trial of potassium paraamino-benzoate (POTABA1) in Peyronie's disease. Progr Reprod Biol Med 9: 61-67.

4. Weidner W, Hauck EW, Schnitker J (2005) Potassium para aminobenzoate (POTABA) in the treatment of Peyronie's disease: a prospective, placebocontrolled, randomized study. Eur Urol 47(4): 530-535.

5. Levine LA, Goldman KE, Greenfield JM (2002) Experience with intraplaque injection of verapamil for Peyronie's disease. J Urol 168: 621-625.

6. Gontero P, Di Marco M, Giubilei G, Bartoletti R, Pappagallo G, et al. (2009) Use of penile extender in the treatment of penile curvature as a result of Peyronie's Desease. Results of a phase II randomized prospective study. J Sex Med 6(2): 558-566.

7. Ralph D, Gonzalez CN, Mirone V, Perovic S, Sohn M, et al. (2010) The management of Peyronie's disease: evidence-based 2010 guidelines. J Sex Med 7(7): 2359-2374.

8. Muller A, Mulhall JP (2009) Peyronie's disease intervention trials: methodological challenges and issues. J Sex Med 6(3): 848-861.

9. Abern MR, Levine LA (2009) Peyronie's disease: evaluation and review of nonsurgical therapy. The Scientific World Journal 9: 665-675. 
10. Jordan GH, Carson CC, Lipshultz LI (2014) Minimally invasive treatment of Peyronie's disease: evidence-based progress. BJU Int 114(1): 16-24.

11. Abern MR, Larsen S, Levine LA (2012) Combination of penile traction, intralesional Verapamil, and oral therapies for Peyronie's Desease. J Sex Med 9(1): 288-295.

12. Assoian RK, Klein EA (2008) Growth control by intracellular tension and extracellular stiffness. Trends Cell Biol 18(7): 347-352.

13. Kueronya V, Miernik A, Stupar S, Kojovic V, Hatzichristodoulou G, et al. (2015) International multicentre psychometric evaluation of patient- reported outcome data for the treatment of Peyronie's disease. BJU Int 115(5): 822-828.

14. Martínez SJI, Egui A, Moncada I, Minaya J, Ballesteros CM, et al. (2014) Acute phase Peyronie's disease management with traction device: a nonrandomized prospective controlled trial with ultrasound correlation. J Sex Med 11(2): 506-515.

15. Pryor JP, Farell CF (1983) Controlled clinical trial of Vitamin E in Peyronie's disease. Prog Reprod Biol 9: 41-45. (c) (i) Creative Commons Attribution 4.0

For possible submissions Click Here

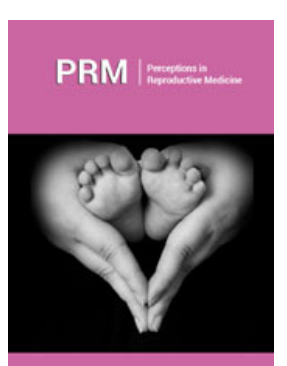

Perceptions in Reproductive Medicine

\section{Benefits of Publishing with us}

- High-level peer review and editorial services

- Freely accessible online immediately upon publication

- Authors retain the copyright to their work

- Licensing it under a Creative Commons license

- Visibility through different online platforms 